\title{
Amitraz and its metabolite modulate honey bee cardiac function and tolerance to viral infection
}

Scott T. O'Neal ${ }^{1}$, Carlyle C. Brewster ${ }^{1}$, Jeffrey R. Bloomquist ${ }^{2}$, Troy D. Anderson ${ }^{3}$

1 Department of Entomology, Virginia Tech, Blacksburg, VA, USA 2 Department of

Entomology and Nematology, Emerging Pathogens Institute, University of Florida, Gainesville, FL, USA 3 Department of Entomology, University of Nebraska, Lincoln, NE, USA

Corresponding Author: Scott T. O’Neal (onealst@vt.edu)

\begin{abstract}
The health and survival of managed honey bee (Apis mellifera) colonies are affected by multiple factors, one of the most important being the interaction between viral pathogens and infestations of the ectoparasitic mite Varroa destructor. Currently, the only effective strategy available for mitigating the impact of viral infections is the chemical control of mite populations. Unfortunately, the use of in-hive acaricides comes at a price, as they can produce sublethal effects that are difficult to quantify, but may ultimately be as damaging as the mites they are used to treat. The goal of this study was to investigate the physiological and immunological effects of the formamidine acaricide amitraz and its primary metabolite in honey bees. Using flock house virus as a model for viral infection, this study found that exposure to a formamidine acaricide may have a negative impact on the ability of honey bees to tolerate viral infection. Furthermore, this work has demonstrated that amitraz and its metabolite significantly alter honey bee cardiac function, most likely through interaction with octopamine receptors. The results suggest a potential drawback to the in-hive use of amitraz and raise intriguing questions about the relationship between insect cardiac function and disease tolerance.
\end{abstract}

\section{Keywords}

Honey bee; heart rate; virus; acaricide; amitraz; DPMF; octopamine; phentolamine

\section{Introduction}


The honey bee (Apis mellifera) is valued for providing economically and agriculturally important pollination services, as well as for providing honey and other natural products. Unacceptably high annual losses in the number of managed bee colonies in the United States (Seitz et al., 2016) have increased public awareness of pollinator health issues and focused research efforts on understanding why these losses occur. Although there exist a wide variety of factors that negatively affect pollinator health (Goulson et al., 2015), one of the most significant threats to the survival of managed bee colonies is the risk of acute viral infections (Evans and Schwarz, 2011; Manley et al., 2015). The growing impact of viral infections is associated with the increased prevalence of the ectoparasitic mite Varroa destructor, which facilitates the spread of viral pathogens and weakens the immune responsiveness of bees, causing previously covert viral infections to become devastating outbreaks (Genersch and Aubert, 2010; Le Conte et al., 2010; Nazzi et al., 2012). At this time, the only effective strategy that exists for minimizing the spread and impact of viral infections is the management of mite infestations, which relies heavily upon the use of apicultural acaricides such as the organophosphate coumaphos (Checkmite $\AA$ ), the pyrethroids tau-fluvalinate (Apistan $\AA$ ) and flumethrin (Bayvarol®), and the formamidine amitraz (Apivar®) (Rosenkranz et al., 2010).

One of the most comprehensive surveys to date of agrochemicals associated with managed bee colonies in the United States found that acaricides used to control Varroa, or their associated metabolites, are among the most ubiquitous contaminants of the hive environment (Mullin et al., 2010). Although the acaricides coumaphos and taufluvalinate have decreased in effectiveness over the years, due to metabolic and targetsite resistance in Varroa populations (Pettis, 2004), they were the most common hive contaminants detected in the survey (Mullin et al., 2010), likely as a result of their continued use by beekeepers and their lipophilic nature, which allows them to persist in beeswax (Bogdanov, 2006). While amitraz does not persist in the hive environment (Martel et al., 2007), its metabolite $N$-(2,4-dimethylphenyl)- $N$ '-methylformamidine (DPMF) does accumulate and was among the ten most commonly detected pesticides in wax, pollen, and the bees themselves (Mullin et al., 2010). This finding is somewhat surprising, as amitraz was withdrawn from commercial use in 1994 and not registered for apicultural use at the time of the survey (Johnson et al., 2010), which suggests that it 
continued to be employed as a control measure in many areas. Since amitraz was reregistered for apicultural use by the Environmental Protection Agency in 2013, it is likely that its presence in the hive environment has increased.

Amitraz is a formamidine acaricide that was originally marketed in the United States under the trade name Miticur®, until it was withdrawn from commercial apicultural use. Amitraz, however, remained available as a veterinary acaricide under the trade name Taktic $\circledast$, which was not labeled for apicultural use, until being reregistered under the name Apivar ${ }^{\circledR}$. Formamidines act as octopaminergic agonists in arthropods (Evans and Gee, 1980), suggesting that they are likely to influence honey bee behavior, learning, and memory formation, in addition to affecting physiological processes related to various tissues and sensory organs (Roeder, 2005). The biogenic monoamine octopamine is understood to act as a neurotransmitter/neuromodulator in insects and other invertebrates, homologous to the noradrenergic system of vertebrates (Roeder, 1999). High levels of octopamine in the brain of honey bee workers can influence the division of labor within the colony (Schulz and Robinson, 2001) and affect foraging behavior (Barron et al., 2007). Stimulation of octopamine receptors improves kin recognition in honey bees (Robinson et al., 1999), and octopamine receptors appear to play a role in modulating honey bee hygienic behavior (Spivak et al., 2003). Octopamine, a known cardioaccelerant in insects, alters heart rate in isolated honey bee hearts (Papaefthimiou and Theophilidis, 2011), and the acaricide amitraz appears to have similar effects in this model (Papaefthimiou et al., 2013). Acute exposure to amitraz has been shown to cause cell death in the midgut of honey bee larvae (Gregorc and Bowen, 2000), but does not appear to affect learning, short-term memory, or hemolymph octopamine levels in honey bee workers (Rix and Cutler, 2017), nor has it been found to affect the survival or sperm viability of honey bee drones (Johnson et al., 2013). Though some acaricides have been found to reduce honey bee immunocompetence (Boncristiani et al., 2012; Locke et al., 2012), amitraz was not observed to alter the expression profiles of a wide range of metabolic genes involved in detoxification, immunity, and development, nor did it appear to increase pathogen levels in treated honey bee colonies (Boncristiani et al., 2012). 
At this time, no studies have been published that characterize the physiological or immunological effects of the amitraz metabolite DPMF in honey bees. Furthermore, little is known about the effect of formamidines, or any other class of pesticides, on the ability of bees to resist or tolerate viral infections. A number of challenges are associated with the study of viral infection in bees, including the high prevalence of covert, and often concurrent, viral infections in managed colonies (Chen et al., 2004; de Miranda et al., 2010; Runckel et al., 2011), as well as a lack of availability of infectious clones of beespecific viruses. These factors pose a challenge for researchers focused on the outcome of infection with a single virus. While some research has been conducted using semi-purified virus preparations (Chen and Siede, 2007), complete removal of contaminating viruses is often impossible, making the accurate characterization of infection dynamics difficult. This represents a significant knowledge gap, given the impact that viruses have on colony health and survival (Cox-Foster et al., 2007; Johnson et al., 2009; McMenamin and Genersch, 2015), the effect of pesticide usage on pollinator health (Mullin et al., 2010), and concerns related to managed bee colony losses (Neumann and Carreck, 2010; Ratnieks and Carreck, 2010). The research described here will begin to address this gap by investigating the effect of amitraz and DPMF on the cardiac function of an agriculturally and economically important pollinator and model social insect. This work will then utilize a recently-described model virus system (O'Neal et al., 2017a) to assess the impact of amitraz and DPMF on the outcome of a viral infection in the honey bee.

\section{Materials and Methods}

\subsection{Subjects}

European honey bees (Apis mellifera) from colonies located at the Virginia Tech Price's Fork Research Facility (Blacksburg, VA) apiary were used for all experiments. Colonies received no pesticide treatments or other exposure to in-hive chemical controls, but otherwise were maintained according to standard beekeeping practices for commercial hives. All bees that were housed in the lab overnight or longer were maintained in incubators at $32{ }^{\circ} \mathrm{C}$ with a relative humidity of $50-80 \%$. For all dissection and heart rate assays, worker bees were collected from brood frames during typical foraging times to 
ensure collection of predominately nurse bees. Any workers collected from the apiary that were housed in the lab incubators overnight were provided ad libitum access to honey and a $50 \%$ solution $(\mathrm{w} / \mathrm{v})$ of sucrose in water. For all survival experiments, frames

of emerging worker brood were removed from the hive and housed in a lab incubator in order to obtain age-matched cohorts of bees. Newly emerged bees were collected from these frames over the course of $24 \mathrm{~h}$ and housed in cages in groups of approximately 25 bees per cage with ad libitum access to a $50 \%$ solution $(w / v)$ of sucrose in water. Cages were maintained in the incubator for the duration of the experiment and were provided with $1 / 4$ portions of a queen mandibular pheromone-impregnated strip (Mann Lake Ltd.) to reduce stress by simulating the presence of an egg-laying queen.

\subsection{Dissection and heart rate assay}

Visualization and pharmacological manipulation of the honey bee heart, as well as measurements of heart rate, were conducted as previously described (O'Neal and Anderson, 2016; O'Neal et al., 2017b). Individual bees were dissected to separate the dorsal abdominal wall and expose the dorsal vessel, which was bathed in an isotonic solution ( $1 / 4$ strength Ringer's solution; Sigma-Aldrich) and given time to allow the heartbeat to stabilize. Baseline heart rate was measured for $1 \mathrm{~min}$ prior to treatment, then measured again 2 min later. All test compounds were dissolved in dimethyl sulfoxide (DMSO) and then diluted in $1 / 4$ strength Ringer's solution to prepare stock solutions. Test compounds were prepared by serial dilution, ensuring a consistent vehicle of $1 \%$ DMSO in $1 / 4$ strength Ringer's solution. Changes in heart rate were reported as percent change relative to the baseline heart rate, measured in beats per minute (BPM).

\subsection{Concentration response experiment}

The cardiomodulatory effects of the formamidine acaricide amitraz and its primary metabolite DPMF on bee heart rate were evaluated by testing a range of concentrations for each compound, along with the insect neurotransmitter/neuromodulator octopamine and the octopamine receptor antagonist phentolamine. All test compounds were obtained from Sigma-Aldrich at the highest purity available and prepared and delivered in $1 \%$ DMSO in $1 / 4$ strength Ringer's solution, which served as the vehicle control. Test 
compounds were evaluated across a range of concentrations spanning the high nanomolar to the low millimolar in order to establish a profile for each compound. The sample size for each treatment group consisted of 10 individual bee dissections.

\subsection{Phentolamine pretreatment experiment}

The ability of amitraz and its metabolite DPMF to modulate honey bee heart rate via interaction with octopamine receptors was examined to determine if phentolamine, a specific octopamine receptor antagonist in insects, including honey bees (Degen et al., 2000), could block their effects. Based on the results of the previous experiment, 100 $\mathrm{nM}$ phentolamine was selected to test against $100 \mu \mathrm{M}$ octopamine, amitraz, and DPMF. Phentolamine was tested at the highest concentration that did not produce a significant effect on heart rate. The concentrations of octopamine, amitraz, and DPMF were selected due to their significant effect on heart rate. The dissection and pharmacological manipulation assay remained unchanged, except that following dissection and visualization, the heart was bathed in either vehicle or vehicle containing $100 \mathrm{nM}$ phentolamine. The protocol then continued as previously described, with the application of $100 \mu \mathrm{M}$ octopamine, amitraz, DPMF, or vehicle control following $1 \mathrm{~min}$ of baseline heart rate assessment. The sample size for each treatment group consisted of 12 individual bee dissections.

\subsection{Acaricide exposure}

Bees were exposed to acaricides either through oral administration or exposure to a portion of an Apivar® Miticide Strip (Mann Lake Ltd.) in the cage. For oral administration, stock solutions of amitraz and DPMF (Sigma-Aldrich) were initially prepared in DMSO. Test groups received $50 \%$ sucrose solution $(\mathrm{w} / \mathrm{v})$ supplemented with either amitraz or DPMF (100 $\mu \mathrm{M}$ final concentration) in DMSO (1\% final concentration), while vehicle control groups received sucrose solution supplemented only with $1 \%$ DMSO. In order to avoid complications related to the poor solubility of amitraz, supplemented solutions were prepared fresh daily. The concentration of amitraz and DPMF $(100 \mu \mathrm{M})$ was selected based on preliminary testing, which revealed this to be the highest concentration of either compound that did not significantly affect bee survival over a $10 \mathrm{~d}$ period. Preliminary testing also demonstrated no significant 
effects of sucrose solution supplemented with $1 \%$ DMSO on bee survival over a $10 \mathrm{~d}$ period. In order to simulate the type of exposure that bees would typically have to an inhive acaricide treatment, test cages were equipped with a $0.5 \mathrm{~cm} \times 4 \mathrm{~cm}$ portion of an amitraz-impregnated acaricide strip (3.33\% active ingredient). Preliminary testing demonstrated that the presence of the acaricide strip did not have any effect on bee survival over a $10 \mathrm{~d}$ period.

\subsection{Viral infection}

Viral infections were performed using flock house virus (FHV), generously provided by Dr. Anette Schneemann (The Scripps Research Institute, La Jolla, California), that was purified as previously described (Marshall and Schneemann, 2001). FHV has been shown to pathogenically infect honey bees and has been used as a model for the study of viral infections in bees (O'Neal et al., 2017a). Viral stocks were prepared in $10 \mathrm{mM}$ Tris- $\mathrm{HCl}, \mathrm{pH}$ 7.5. Infections were performed by injection (Nanoject II apparatus; Drummond Scientific) of $50.6 \mathrm{nl}$ of a $2 \times 10^{7}$ plaque-forming units $(\mathrm{pfu}) / \mu \mathrm{l}$ viral suspension into the thorax of each bee, resulting in the delivery of $1 \times 10^{6}$ pfu of FHV/bee. This virus titer was selected based on previous work (O'Neal et al., 2017a), which suggested that it would produce a moderate infection that would permit the observation of changes in survival over time in different treatment groups. Injection of the same volume of $10 \mathrm{mM}$ Tris- $\mathrm{HCl}, \mathrm{pH} 7.5$, was used as a vehicle control. Preliminary testing compared the survival of bees following vehicle control injections and sham injections, in which the bee thorax was punctured by the needle without delivery of fluid, and found that neither group experienced significant changes in survival relative to uninjected bees over a $10 \mathrm{~d}$ period.

\subsection{Oral dosing survival experiment}

The effects of amitraz and its metabolite DPMF on the survival of virus-challenged honey bees was examined by orally exposing individuals to these compounds and then infecting them with FHV. For all survival experiments, six replicates of 25 bees each were used for each treatment group. Bees were injected with either vehicle or virus following $24 \mathrm{~h}$ of exposure to amitraz-supplemented, DPMF-supplemented, or DMSOsupplemented sucrose solution as the only source of food and water. Bees were 
provided access to the same sucrose solution, which was prepared fresh each day, for the duration of the test. Survival was observed daily following injection for $10 \mathrm{~d}$.

\subsection{Acaricide strip survival experiment}

The effects of contact exposure to amitraz on the survival of virus-challenged honey bees was examined by exposing bees to an amitraz-impregnated plastic strip, a commonly-used treatment in mite-infested hives, and then infecting them with FHV. As in the previous survival experiments, six replicates of 25 bees each were used for each treatment group. Bees were injected with either vehicle or virus following $24 \mathrm{~h}$ of exposure to the amitraz strip and remained exposed to the strip throughout the duration of the test. Survival was observed daily following injection for $5 \mathrm{~d}$.

\subsection{Statistical Analysis}

All heart rate assay analyses and calculations were conducted using GraphPad Prism 7 (GraphPad Software, Inc., La Jolla, CA). All heart rate assay results are expressed as the mean \pm standard deviation (SD). Each heart rate assay treatment group was subjected to a D'Agostino-Pearson test for normality $(P<0.05)$ and not all groups were found to come from a normally distributed population; therefore, a nonparametric, twotailed Mann-Whitney test $(P<0.05)$ was used for all comparisons between heart rate assay treatment groups. A one-way analysis of variance (ANOVA) was used to test for differences $(P<0.05)$ in the mean baseline heart rate of groups in the concentration response experiment. All survival experiment results are reported as Kaplan-Meier survival curves, calculated using GraphPad Prism 7, with significant differences between the survival curves determined by the log-rank (Mantel-Cox) test. For the acaricide strip survival experiment, the treatment mortality (Untreated/Virus and Amitraz/Virus) was corrected for control mortality (Untreated/Vehicle and Amitraz/Vehicle) using Abbott's formula (Abbott, 1925). A mixed model repeatedmeasures analysis of the corrected mortality data was performed as previously described (Singh et al., 2016) using JMP Version 13 (SAS Institute Inc., Cary, NC) to determine differences between treatments and their interaction with time. In the model, treatment, day, and treatment by day were the fixed effects and replicate was the random effect. 


\section{Results}

\subsection{Concentration response experiment}

Phentolamine treatment decreased honey bee heart rate, whereas octopamine, amitraz, and DPMF treatment increased heart rate (Figure 1). Phentolamine produced a concentration-dependent effect on heart rate, as the application of increasing concentrations resulted in greater decreases in heart rate with no evidence of an increase at any concentration. A significant decrease in heart rate relative to the vehicle control was observed at concentrations of $300 \mathrm{nM}$ and above (Mann-Whitney test; $P<$ 0.01 ) with maximal effect (complete cessation of heart beat) observed at $1 \mathrm{mM}$. Mean baseline heart rate $( \pm S D)$ across phentolamine treatment groups was $105.0 \pm 2.5 \mathrm{BPM}$. Octopamine significantly increased heart rate relative to the vehicle control at concentrations of $3 \mu \mathrm{M}$ and above (Mann-Whitney test; $P<0.01$ ). Mean baseline heart rate $( \pm \mathrm{SD})$ across octopamine treatment groups was $101.7 \pm 9.6$ BPM. Amitraz significantly increased heart rate relative to the vehicle control at concentrations of 30 $\mu \mathrm{M}$ and above (Mann-Whitney test; $P<0.01$ ). Mean baseline heart rate $( \pm \mathrm{SD})$ across amitraz treatment groups was $102.8 \pm 4.7$ BPM. DPMF significantly increased heart rate relative to the vehicle control at concentrations of $1 \mu \mathrm{M}$ and above (Mann-Whitney test; $P<0.01)$. Mean baseline heart rate $( \pm S D)$ across DPMF treatment groups was $105.4 \pm$ 3.1 BPM. Maximal effects could not be determined for octopamine, amitraz, and DPMF due to solubility issues at concentrations greater than $1 \mathrm{mM}$. No statistically significant differences were detected between the mean baseline heart rates of each experimental group.

\subsection{Phentolamine pretreatment experiment}

Pretreatment with phentolamine uniformly blocked the cardioacceleratory effects of octopamine, amitraz, and DPMF (Figure 2). Pretreatment with $100 \mathrm{nM}$ phentolamine followed by treatment with vehicle resulted in no significant change in heart rate relative to the vehicle control. Pretreatment with vehicle followed by $100 \mu \mathrm{M}$ octopamine, 100 $\mu \mathrm{M}$ amitraz, or $100 \mu \mathrm{M}$ DPMF resulted in a significant increase in heart rate (MannWhitney test; $P<0.001$ ), with effects comparable to what was observed in the previous experiment (Figure 1). Pretreatment with $100 \mathrm{nM}$ phentolamine followed by treatment 
with $100 \mu \mathrm{M}$ octopamine, $100 \mu \mathrm{M}$ amitraz, or $100 \mu \mathrm{M}$ DPMF resulted in a complete loss of effect, with the observed heart rate not significantly different from vehicle treatment, but significantly reduced when compared to the corresponding drug-treated replicates without phentolamine (Mann-Whitney test; $P<0.01$ ). Mean baseline heart rate $( \pm S D)$ across treatment groups measured $98.0 \pm 2.7$ BPM.

\subsection{Oral dosing survival experiment}

Treatment with amitraz and the amitraz metabolite DPMF decreased the survival of honey bees following infection with $1 \times 10^{6} \mathrm{pfu}$ of $\mathrm{FHV} / \mathrm{bee}$, relative to the infected control group (Figure 3). Bees in the uninfected control groups all experienced approximately $10 \%$ mortality by $5 \mathrm{~d}$ post-injection and $25 \%$ mortality by the end of the study at $10 \mathrm{~d}$ post-injection. Infected controls experienced $46 \%$ mortality by $5 \mathrm{~d}$ postinjection and reached $100 \%$ mortality at $9 \mathrm{~d}$ post-injection, whereas infected bees receiving amitraz treatment experienced $83 \%$ mortality by $5 \mathrm{~d}$ post-injection and reached $100 \%$ mortality at $7 \mathrm{~d}$ post-injection. Similarly, infected bees receiving DPMF treatment experienced $75 \%$ mortality by 5 d post-injection and reached $100 \%$ mortality at $7 \mathrm{~d}$ post-injection. Log-rank tests of the Kaplan-Meier survival curves indicated a significant difference in survival between infected bees that were treated with amitraz and infected bees that were treated with vehicle $\left(x^{2}=54.32\right.$; $\left.d f=1 ; P<0.0001\right)$. Similarly, a significant difference in survival was also detected between infected bees that were treated with DPMF and infected bees that were treated with vehicle $\left(x^{2}=\right.$ 41.45; $\mathrm{df}=1 ; P<0.0001)$. There was no significant difference in survival detected between the uninfected control groups treated with vehicle, amitraz, or DPMF, nor was there any difference between the infected groups treated with amitraz and DPMF.

\subsection{Acaricide strip survival experiment}

Exposure to amitraz-impregnated plastic strips decreased the survival of bees challenged with virus and vehicle injections alike, relative to the respective control groups (Figure 4). Infected bees exposed to amitraz experienced $85 \%$ mortality just $1 \mathrm{~d}$ after infection, compared to only $10 \%$ for the infected control group. Bees injected with vehicle and exposed to amitraz experienced $32 \%$ mortality after just $1 \mathrm{~d}$, compared to only $1 \%$ mortality for the uninfected control group. Infected bees exposed to amitraz 
experienced $100 \%$ mortality by $3 \mathrm{~d}$ after injection, whereas the curve leveled off for bees injected with vehicle and exposed to amitraz starting at $2 \mathrm{~d}$ after injection and their survival remained consistent at approximately $40 \%$ mortality for the remainder of the study. Log-rank tests of the Kaplan-Meier survival curves indicated a significant difference in survival between the Untreated/Vehicle and Untreated/Virus groups $\left(x^{2}=\right.$ 44.43; $\mathrm{df}=1 ; P<0.0001$ ), between the Untreated/Vehicle and Amitraz/Vehicle groups $\left(X^{2}=43.57 ; \mathrm{df}=1 ; P<0.0001\right)$, and between the Untreated/Vehicle and Amitraz/Virus groups $\left(X^{2}=329.20 ; \mathrm{df}=1 ; P<0.0001\right)$. Significant differences were also detected between the Untreated/Virus and Amitraz/Virus groups $\left(X^{2}=240.70 ; \mathrm{df}=1 ; P<0.0001\right)$ and between the Amitraz/Vehicle and Amitraz/Virus groups $\left(X^{2}=147.90 ; \mathrm{df}=1 ; P<\right.$ 0.0001), but no difference was detected between the Untreated/Virus and Amitraz/Vehicle groups. When the treatment mortality was corrected for the control mortality, amitraz was found to have a significant effect on the survival of virus-infected bees $(F=1997.39 ; \mathrm{df}=1,13 ; P<0.0001)$.

\section{Discussion}

This work has demonstrated that the formamidine acaricide amitraz and its metabolite DPMF significantly alter honey bee cardiac function, most likely through interaction with octopamine receptors. Treatment with the octopamine receptor antagonist phentolamine decreased honey bee heart rate, whereas treatment with octopamine, amitraz, and DPMF increased heart rate. Furthermore, pretreatment with a low concentration of phentolamine was found to block the effects of octopamine, amitraz and DPMF. This work has also demonstrated that exposure to a formamidine acaricide may have a negative impact on the ability of honey bees to tolerate viral infection. Oral treatment with amitraz and DPMF similarly decreased the survival of virus-challenged bees, as did exposure to amitraz-impregnated plastic strips routinely used in the treatment of mite-infested hives. These findings are significant as they highlight the complex nature of insect immunity and disease tolerance, as well as the interrelatedness of diverse physiological systems.

The changes in heart rate observed with this approach are comparable in many respects to those observed using other methods, most notably two studies that tested 
the effects of octopamine, phentolamine, and amitraz on ex vivo honey bee heart preparations using a force-displacement transducer and intracellular recordings of heart muscle fibers using microelectrodes (Papaefthimiou et al., 2013; Papaefthimiou and Theophilidis, 2011). In these studies, octopamine was observed to have a cardioacceleratory effect in bees at concentrations in the picomolar range and above, while phentolamine blocked contractions in the micromolar range (Papaefthimiou and Theophilidis, 2011). Amitraz was also observed to have a cardioacceleratory effect on ex vivo bee hearts at concentrations in the nanomolar and micromolar ranges and above, as well as in experiments examining in vivo effects via injection or oral administration (Papaefthimiou et al., 2013). These studies, however, reported biphasic effects of both octopamine and amitraz, noting that at lower concentrations, both compounds could have inhibitory effects. Octopamine was reported to act as an antagonist in the femtomolar range (Papaefthimiou and Theophilidis, 2011), suggesting extreme sensitivity of the bee heart, though even the authors acknowledge that this might be unrealistically low. Amitraz was observed to have inhibitory effects on bee cardiac activity at concentrations in the picomolar range, while at higher concentrations, an initial inhibitory response preceded the observed cardioacceleration (Papaefthimiou et al., 2013).

The biphasic effects and the extremely high sensitivity detected in these two studies may be due in part to the significant differences between the recording methods used in these studies and the work reported here. Another significant difference, however, is that both of these studies reported that recordings of ex vivo heart preparations did not begin until an hour or more following dissection, during which time significant changes in bee heart rate were reported to take place (Papaefthimiou and Theophilidis, 2011), resulting in the development of a bursting pattern that appears quite different from the steady heart rate observed using the method reported here. Although the dissection and visualization method employed in this work (O'Neal and Anderson, 2016) has been observed to allow heart preparations to continue beating for two hours or more, provided periodic renewal of the isotonic solution bathing the heart, qualitative differences suggesting tissue degradation can be observed, in addition to slowing of heart rate and localized or partial loss of heart function. To avoid this, observations of 
heart rate typically began within approximately $5 \mathrm{~min}$ of dissection, which provided sufficient time for the heart rate to stabilize. Consequently, these differences in the observed effects of octopamine and amitraz are likely due to the significant differences in methodology employed. As DPMF has not been previously tested in a similar model, no such comparisons can be made.

Exposure to cardiomodulatory compounds such as amitraz can have a significant impact on a variety of physiological processes, as the insect circulatory system is understood to play a role in thermoregulation, ventilation, and the maintenance of homeostasis. Several studies have also found that insect circulatory and immune systems can closely interact to regulate infections (King and Hillyer, 2012; Sigle and Hillyer, 2016), reinforcing the idea that there exists an extensive level of integration between cardiac function and the insect immune response. The primary insect antiviral immune response is the post-transcriptional gene silencing mechanism known as RNA interference (RNAi), which initiates targeted degradation of RNA in response to the presence of double-stranded RNA (dsRNA) (Ding, 2010; Ding and Voinnet, 2007). While bees have been shown to employ an antiviral RNAi response (Chejanovsky et al., 2014; Desai et al., 2012; Maori et al., 2009; Wang et al., 2013), there is also evidence that the presence of nonspecific dsRNA is sufficient to reduce virus production in bees (Flenniken and Andino, 2013). Another difference noted during a comprehensive examination of honey bee immune responses is that bees were found to only express about one third as many genes associated with insect immunity as have been observed in fruit flies and mosquitoes (Evans et al., 2006). One explanation for this discrepancy could be the effectiveness of colony-level, social immune barriers to infection. Another possibility is that bees also rely on tissue-specific, homeostatic mechanisms to simply tolerate infection (Schneider and Ayres, 2008). This idea is supported by findings that demonstrate an essential role for the evolutionarily conserved cardiac ion channel known as the ATP-sensitive inwardly rectifying potassium ( $\left.\mathrm{K}_{\mathrm{ATP}}\right)$ channel in the resistance to infection by a cardiotropic virus in Drosophila, through modulation of RNAi (Eleftherianos et al., 2011). More recent work has provided evidence of $\mathrm{K}_{\text {ATP }}$ channel regulation of viral infections in bees (O'Neal et al., 2017a) and proposed an important 
role for this ion channel in connecting the antiviral immune response of bees to changes in cellular metabolism induced by exposure to environmental stressors.

It is understood that exposure to chronic stress gradually weakens the immune response and reduces the metabolic activity of an organism until it is no longer able to survive (McEwen, 2000). This holds true for bees as well, since physiological stress can have a wide range of detrimental consequences for bee health and survival (Even et al., 2012). Harmful synergistic interactions between simultaneous exposure to pesticides, dietary toxins, and pathogens have been demonstrated in bees, though the specific mechanisms that explain these interactions have yet to be revealed (Alaux et al., 2010; Aufauvre et al., 2012; Kohler et al., 2012; Vidau et al., 2011). Especially relevant to managed colony health is the evidence that apicultural pesticides can negatively impact bee immunity, as the acaricide tau-fluvalinate has been shown to have an effect on host susceptibility to viral infection (Locke et al., 2012), while the acaricides thymol, coumaphos, and formic acid were found to alter the expression of genes related to immunity, detoxification, and development (Boncristiani et al., 2012). The work presented here provides the first evidence that the formamidine acaricide amitraz, as well as its primary metabolite DPMF, may have a negative impact on honey bee antiviral resistance or tolerance to viral infection. This work also demonstrates the physiological effect that amitraz and DPMF can have on bee cardiac function, describing a cardioacceleratory role for both.

Although these findings do not provide direct evidence of a relationship between octopamine receptor-mediated modulation of bee cardiac function and weakened immunity or tolerance to viral infection, it is reasonable to hypothesize that such a relationship could exist, given the integration between insect immune and circulatory systems, as well as the important role of cardiac function in maintaining homeostasis. These findings demonstrate that DPMF is at least as cardioactive as the parent compound amitraz and appears to function in the same manner as amitraz, as the activity of each is blocked by the octopamine receptor antagonist phentolamine. This is important to note, as amitraz is quickly metabolized, but DPMF is less easily degraded and is one of the most commonly encountered agrochemical contaminants in the hive 
environment, as well as the bees themselves (Mullin et al., 2010). This means that managed bees may experience long-term exposure to residual DPMF present in the hive, even after amitraz treatment has been discontinued. This is significant, given that this study reveals a potentially harmful synergistic relationship between exposure to amitraz, as well as DPMF, and the ability to resist or tolerate a viral infection. Although these findings are significant and dramatic, it can be argued that this experimental approach does not truly reflect the typical exposure of bees during an in-hive treatment with amitraz.

In order to simulate in-hive amitraz treatment, caged bees were exposed to small portions of a commercial miticide strip impregnated with amitraz. While preliminary testing found that this type of exposure had no effect on the survival of caged bees, this treatment did have a significant effect on the survival of bees challenged with virus, as well as bees in the control group that were challenged with a vehicle injection. The effects are quite pronounced after just $1 \mathrm{~d}$ post-injection, as there was considerable mortality in both the amitraz-treated groups. Interestingly, mortality levels off at that point in the amitraz-treated group that received a vehicle challenge, whereas the rate of mortality continues in both virus-challenged groups. It is very likely that this observation is related to the age of the bees, which had eclosed between 24 and $48 \mathrm{~h}$ prior to injection. One possibility is that the cuticular layers were still in a state of transition following eclosion, making the cuticle more susceptible to penetration by amitraz during this state (Noble-Nesbitt, 1970). Another possibility is that cuticular injury from the injection also facilitated amitraz penetration. In either case, however, there appears to be a synergistic interaction between exposure to amitraz and either the vehicle or the injury from the injection, which could imply that there is a trade-off, or a competition for resources, that limits the ability of the bee to both detoxify the acaricide and respond to the injury. This interaction warrants a more thorough examination.

These findings have some immediate implications for apiculture practices, as there is likely a trade-off to be considered when the decision is being made to treat a miteinfested hive with amitraz. Unfortunately, the data provided here are insufficient for determining when the economic benefits of treating with amitraz outweigh the possible 
drawbacks of reducing immune responsiveness. More research is needed to determine the practical implications of the interactions between amitraz, DPMF, and honey bee resistance or tolerance to viral infection at the colony level, but the evidence supports a policy of minimizing chemical interventions within the hive. These findings also raise intriguing questions about the nature of this interaction. Is this relationship the result of octopaminergic interference with cardiac function, thereby resulting in a loss of homeostasis and the ability to tolerate infection? Or, do these compounds act through some unknown mechanism to regulate the innate immune response of the insect? Furthermore, are there age-dependent effects that must also be considered here? The answers to these questions would provide significant insight into honey bee physiology and represent a promising area for future research intended to improve understanding of honey bee antiviral immunity, disease tolerance, and their relationship to factors that negatively impact pollinator health.

\section{Acknowledgments}

Jennifer R. Williams for her technical assistance. Dr. Anette Schneemann for providing FHV. This material is based upon work that is supported by the National Institute of Food and Agriculture, United States Department of Agriculture, under award number 2017-67011-26048. The funders had no role in study design, data collection and analysis, decision to publish, or preparation of the manuscript.

\section{Author Contributions}

Conceptualization: STO, JRB, TDA. Investigation: STO. Formal Analysis: STO, CCB, TDA. Original Draft Preparation: STO. Review and Editing: STO, CCB, JRB, TDA.

\section{Author competing interests}

The authors declare no competing financial interests.

\section{References}

Abbott, W. S., 1925. A method of computing the effectiveness of an insecticide. Journal of Economic Entomology. 18, 265-267. 
Alaux, C., et al., 2010. Interactions between Nosema microspores and a neonicotinoid weaken honeybees (Apis mellifera). Environmental Microbiology. 12, 774-782.

Aufauvre, J., et al., 2012. Parasite-insecticide interactions: a case study of Nosema ceranae and fipronil synergy on honeybee. Sci Rep. 2, 326.

Barron, A. B., et al., 2007. Octopamine modulates honey bee dance behavior. Proceedings of the National Academy of Sciences. 104, 1703-1707.

Bogdanov, S., 2006. Contaminants of bee products. Apidologie. 37, 1-18.

Boncristiani, H., et al., 2012. Direct effect of acaricides on pathogen loads and gene expression levels in honey bees Apis mellifera. Journal of Insect Physiology. 58, 613-620.

Chejanovsky, N., et al., 2014. Characterization of viral siRNA populations in honey bee colony collapse disorder. Virology. 454-455, 176-83.

Chen, Y., et al., 2004. Multiple virus infections in the honey bee and genome divergence of honey bee viruses. J Invertebr Pathol. 87, 84-93.

Chen, Y. P., Siede, R., 2007. Honey bee viruses. Adv Virus Res. 70, 33-80.

Cox-Foster, D. L., et al., 2007. A metagenomic survey of microbes in honey bee colony collapse disorder. Science. 318, 283-7.

de Miranda, J. R., et al., 2010. The Acute bee paralysis virus-Kashmir bee virus-Israeli acute paralysis virus complex. J Invertebr Pathol. 103 Suppl 1, S30-47.

Degen, J., et al., 2000. Octopamine receptors in the honey bee and locust nervous system: pharmacological similarities between homologous receptors of distantly related species. British Journal of Pharmacology. 130, 587-594.

Desai, S. D., et al., 2012. Reduction in deformed wing virus infection in larval and adult honey bees (Apis mellifera L.) by double-stranded RNA ingestion. Insect Mol Biol. 21, 446-55. 
Ding, S. W., 2010. RNA-based antiviral immunity. Nat Rev Immunol. 10, 632-44.

Ding, S. W., Voinnet, O., 2007. Antiviral immunity directed by small RNAs. Cell. 130, 413-26.

Eleftherianos, I., et al., 2011. ATP-sensitive potassium channel (K(ATP))-dependent regulation of cardiotropic viral infections. Proc Natl Acad Sci U S A. 108, 120249.

Evans, J. D., et al., 2006. Immune pathways and defence mechanisms in honey bees Apis mellifera. Insect Mol Biol. 15, 645-56.

Evans, J. D., Schwarz, R. S., 2011. Bees brought to their knees: microbes affecting honey bee health. Trends Microbiol. 19, 614-20.

Evans, P. D., Gee, J. D., 1980. Action of formamidine pesticides on octopamine receptors. Nature. 287, 60-2.

Even, N., et al., 2012. General stress responses in the honey bee. Insects. 3, 1271-98.

Flenniken, M. L., Andino, R., 2013. Non-specific dsRNA-mediated antiviral response in the honey bee. PLoS One. 8, e77263.

Genersch, E., Aubert, M., 2010. Emerging and re-emerging viruses of the honey bee (Apis mellifera L.). Vet Res. 41, 54.

Goulson, D., et al., 2015. Bee declines driven by combined stress from parasites, pesticides, and lack of flowers. Science. 347, 1255957.

Gregorc, A., Bowen, I. D., 2000. Histochemical characterization of cell death in honeybee larvae midgut after treatment with Paenibacillus larvae, amitraz, and oxytetracycline. Cell Biology International. 24, 319-324.

Johnson, R. M., et al., 2013. Effect of in-hive miticides on drone honey bee survival and sperm viability. Journal of Apicultural Research. 52, 88-95. 
Johnson, R. M., et al., 2010. Pesticides and honey bee toxicity - USA. Apidologie. 41, 312-331.

Johnson, R. M., et al., 2009. Changes in transcript abundance relating to colony collapse disorder in honey bees (Apis mellifera). Proceedings of the National Academy of Sciences of the United States of America. 106, 14790-14795.

King, J. G., Hillyer, J. F., 2012. Infection-induced interaction between the mosquito circulatory and immune systems. PLoS Pathog. 8, e1003058.

Kohler, A., et al., 2012. Simultaneous stressors: interactive effects of an immune challenge and dietary toxin can be detrimental to honeybees. J Insect Physiol. $58,918-23$.

Le Conte, Y., et al., 2010. Varroa mites and honey bee health: can Varroa explain part of the colony losses? Apidologie. 41, 353-363.

Locke, B., et al., 2012. Acaricide treatment affects viral dynamics in Varroa destructorinfested honey bee colonies via both host physiology and mite control. Appl Environ Microbiol. 78, 227-35.

Manley, R., et al., 2015. Emerging viral disease risk to pollinating insects: ecological, evolutionary and anthropogenic factors. J Appl Ecol. 52, 331-340.

Maori, E., et al., 2009. IAPV, a bee-affecting virus associated with Colony Collapse Disorder can be silenced by dsRNA ingestion. Insect Mol Biol. 18, 55-60.

Marshall, D., Schneemann, A., 2001. Specific packaging of nodaviral RNA2 requires the $\mathrm{N}$-terminus of the capsid protein. Virology. 285, 165-75.

Martel, A.-C., et al., 2007. Acaricide residues in honey and wax after treatment of honey bee colonies with Apivar ${ }^{\circledR}$ or Asuntol $\AA^{3} 50$. Apidologie. 38, 534-544.

McEwen, B. S., 2000. The neurobiology of stress: from serendipity to clinical relevance. Brain Res. 886, 172-189. 
McMenamin, A. J., Genersch, E., 2015. Honey bee colony losses and associated viruses. Current Opinion in Insect Science.

Mullin, C. A., et al., 2010. High levels of miticides and agrochemicals in North American apiaries: implications for honey bee health. PLoS One. 5, e9754.

Nazzi, F., et al., 2012. Synergistic parasite-pathogen interactions mediated by host immunity can drive the collapse of honeybee colonies. PLoS Pathog. 8, e1002735.

Neumann, P., Carreck, N. L., 2010. Honey bee colony losses. Journal of Apicultural Research. 49, 1-6.

Noble-Nesbitt, J., 1970. Structural aspects of penetration through insect cuticles. Pesticide Science. 1, 204-208.

O'Neal, S. T., Anderson, T. D., 2016. Dissection and observation of honey bee dorsal vessel for studies of cardiac function. J Vis Exp. e55029.

O'Neal, S. T., et al., 2017a. ATP-sensitive inwardly rectifying potassium channel regulation of viral infections in honey bees. Scientific Reports.

O'Neal, S. T., et al., 2017b. ATP-sensitive inwardly rectifying potassium channel modulators alter cardiac function in honey bees. Journal of Insect Physiology. 99, 95-100.

Papaefthimiou, C., et al., 2013. Biphasic responses of the honeybee heart to nanomolar concentrations of amitraz. Pesticide Biochemistry and Physiology. 107, 132-137.

Papaefthimiou, C., Theophilidis, G., 2011. Octopamine--a single modulator with double action on the heart of two insect species (Apis mellifera macedonica and Bactrocera oleae): Acceleration vs. inhibition. J Insect Physiol. 57, 316-25.

Pettis, J. S., 2004. A scientific note on Varroa destructor resistance to coumaphos in the United States. Apidologie. 35, 91-92. 
Ratnieks, F. L., Carreck, N. L., 2010. Clarity on honey bee collapse? Science. 327, 1523.

Rix, R. R., Cutler, G. C., 2017. Acute exposure to worst-case concentrations of amitraz does not affect honey bee learning, short-term memory, or hemolymph octopamine levels. Journal of Economic Entomology. 110, 127-132.

Robinson, G. E., et al., 1999. Neurochemicals aid bee nestmate recognition. Nature. 399, 534-535.

Roeder, T., 1999. Octopamine in invertebrates. Prog Neurobiol. 59, 533-61.

Roeder, T., 2005. Tyramine and octopamine: ruling behavior and metabolism. Annu Rev Entomol. 50, 447-77.

Rosenkranz, P., et al., 2010. Biology and control of Varroa destructor. J Invertebr Pathol. 103 Suppl 1, S96-119.

Runckel, C., et al., 2011. Temporal analysis of the honey bee microbiome reveals four novel viruses and seasonal prevalence of known viruses, Nosema, and Crithidia. PLoS One. 6, e20656.

Schneider, D. S., Ayres, J. S., 2008. Two ways to survive infection: what resistance and tolerance can teach us about treating infectious diseases. Nat Rev Immunol. 8, 889-95.

Schulz, D. J., Robinson, G. E., 2001. Octopamine influences division of labor in honey bee colonies. Journal of Comparative Physiology A. 187, 53-61.

Seitz, N., et al., 2016. A national survey of managed honey bee 2014-2015 annual colony losses in the USA. Journal of Apicultural Research. 54, 292-304.

Sigle, L. T., Hillyer, J. F., 2016. Mosquito hemocytes preferentially aggregate and phagocytose pathogens in the periostial regions of the heart that experience the most hemolymph flow. Dev Comp Immunol. 55, 90-101. 
Singh, N., et al., 2016. Posttreatment feeding affects mortality of bed bugs (Hemiptera: Cimicidae) exposed to insecticides. Journal of Economic Entomology. 109, 273283.

Spivak, M., et al., 2003. Hygienic behavior in the honey bee (Apis mellifera L.) and the modulatory role of octopamine. Journal of Neurobiology. 55, 341-354.

Vidau, C., et al., 2011. Exposure to sublethal doses of fipronil and thiacloprid highly increases mortality of honeybees previously infected by Nosema ceranae. PLoS One. 6, e21550.

Wang, H., et al., 2013. Sequence recombination and conservation of Varroa destructor virus-1 and deformed wing virus in field collected honey bees (Apis mellifera). PLoS One. 8, e74508. 

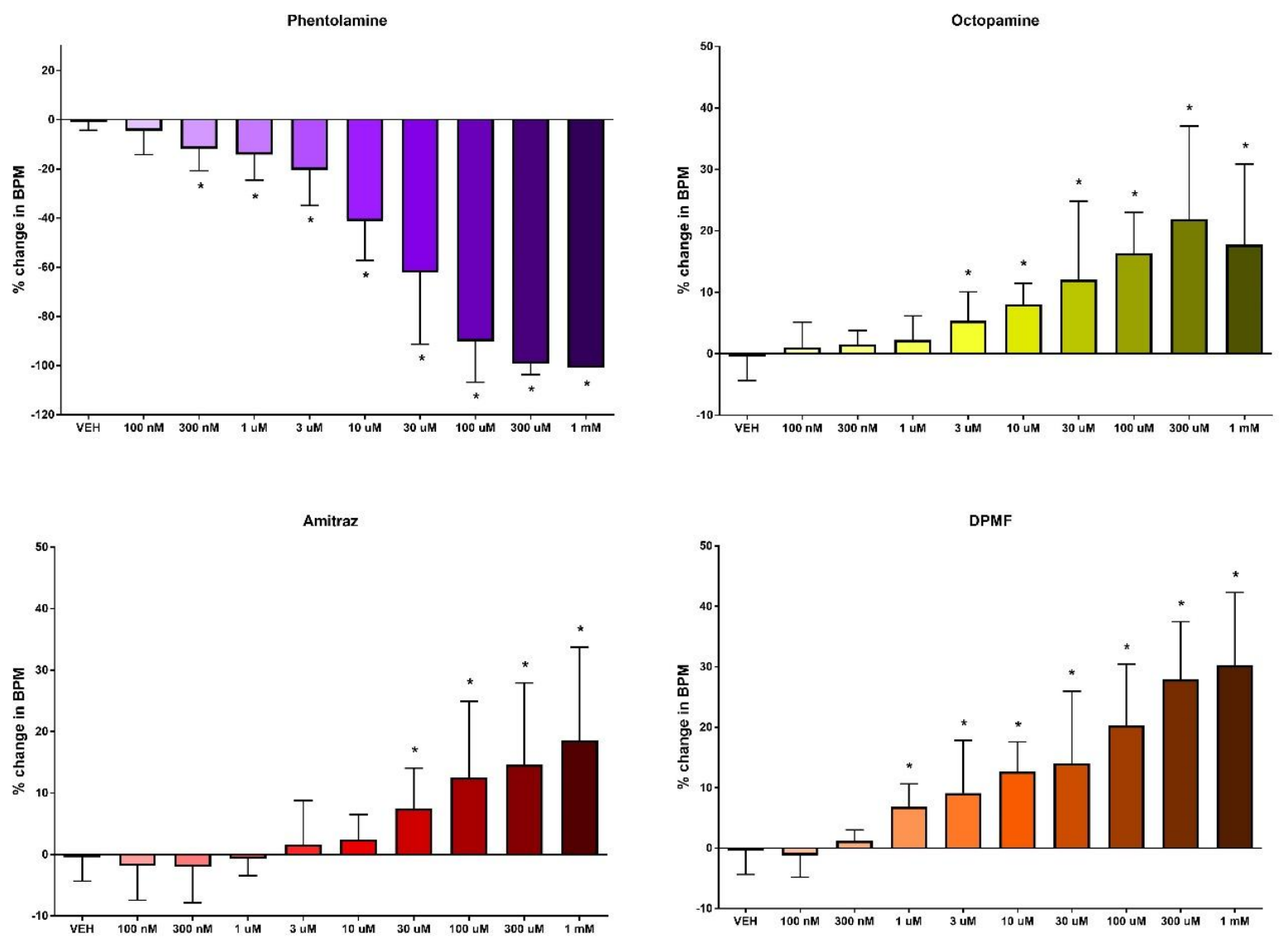

Figure 1. Percent change in heart rate (beats per minute, BPM) resulting from the application of increasing concentrations of amitraz, DPMF, octopamine, and phentolamine. Bars represent mean change in heartbeat frequency \pm standard deviation relative to baseline heart rate $(n=10)$. The mean treatment values were compared to a vehicle control using a nonparametric Mann-Whitney test where $P<0.05$ was considered significant, as represented by *. Data were analyzed using GraphPad Prism 7 software. 


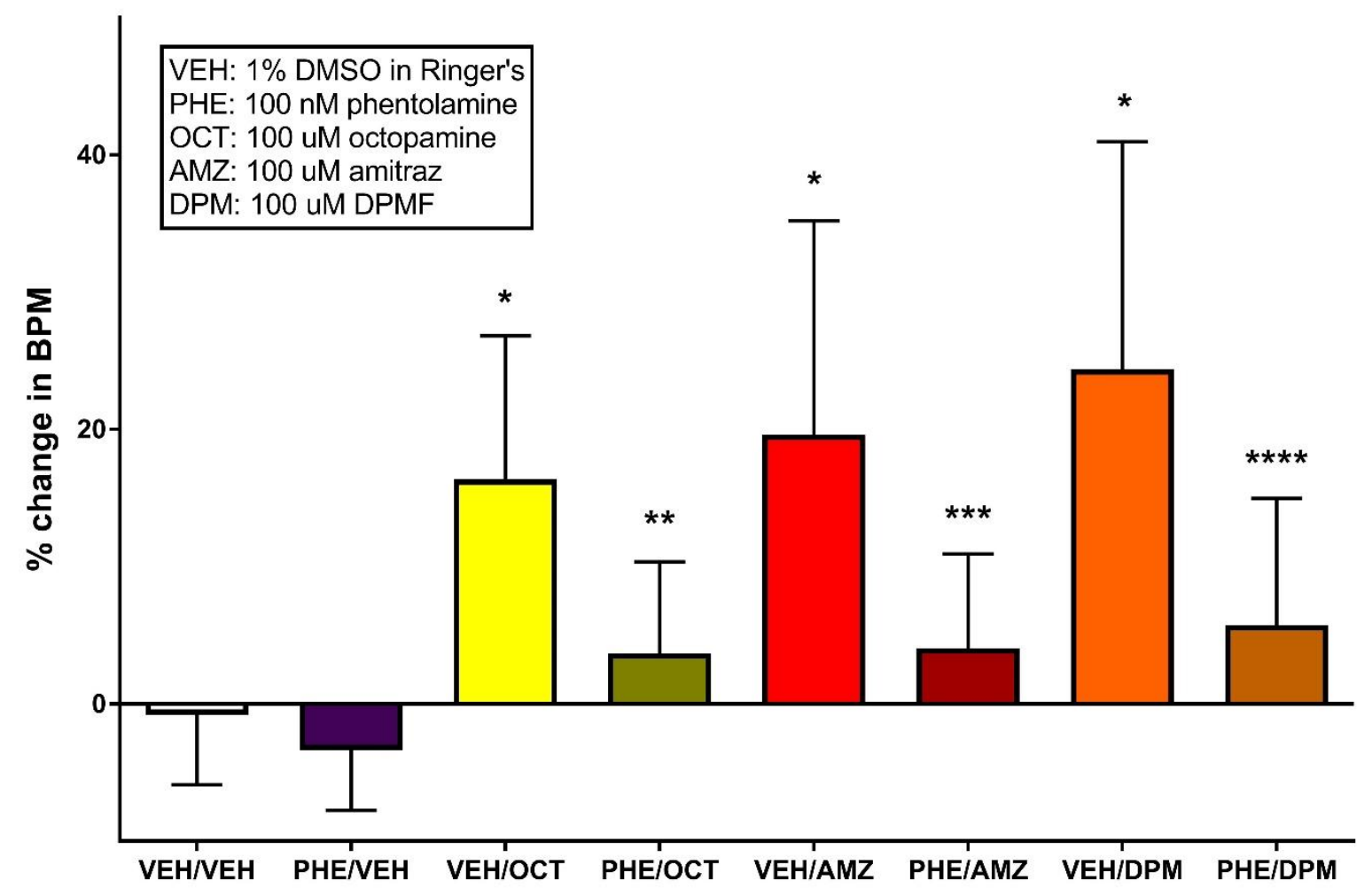

Figure 2. Percent change in heart rate (beats per minute, BPM) resulting from the application of phentolamine followed by octopamine, amitraz, or DPMF. Bars represent mean change in heartbeat frequency \pm standard deviation relative to baseline heart rate $(n=12)$. The mean values were compared using a nonparametric MannWhitney test where $P<0.05$ was considered significant. ${ }^{*}$ indicates a significant difference from VEH/VEH group. ${ }^{* *}$ indicates no significant difference from $V E H / V E H$, but a significant difference from VEH/OCT. ${ }^{* * *}$ indicates no significant difference from $\mathrm{VEH} / \mathrm{VEH}$, but a significant difference from VEH/AMZ. ${ }^{* * * *}$ indicates no significant difference from VEH/VEH, but a significant difference from VEH/DPM. Data were analyzed using GraphPad Prism 7 software. 


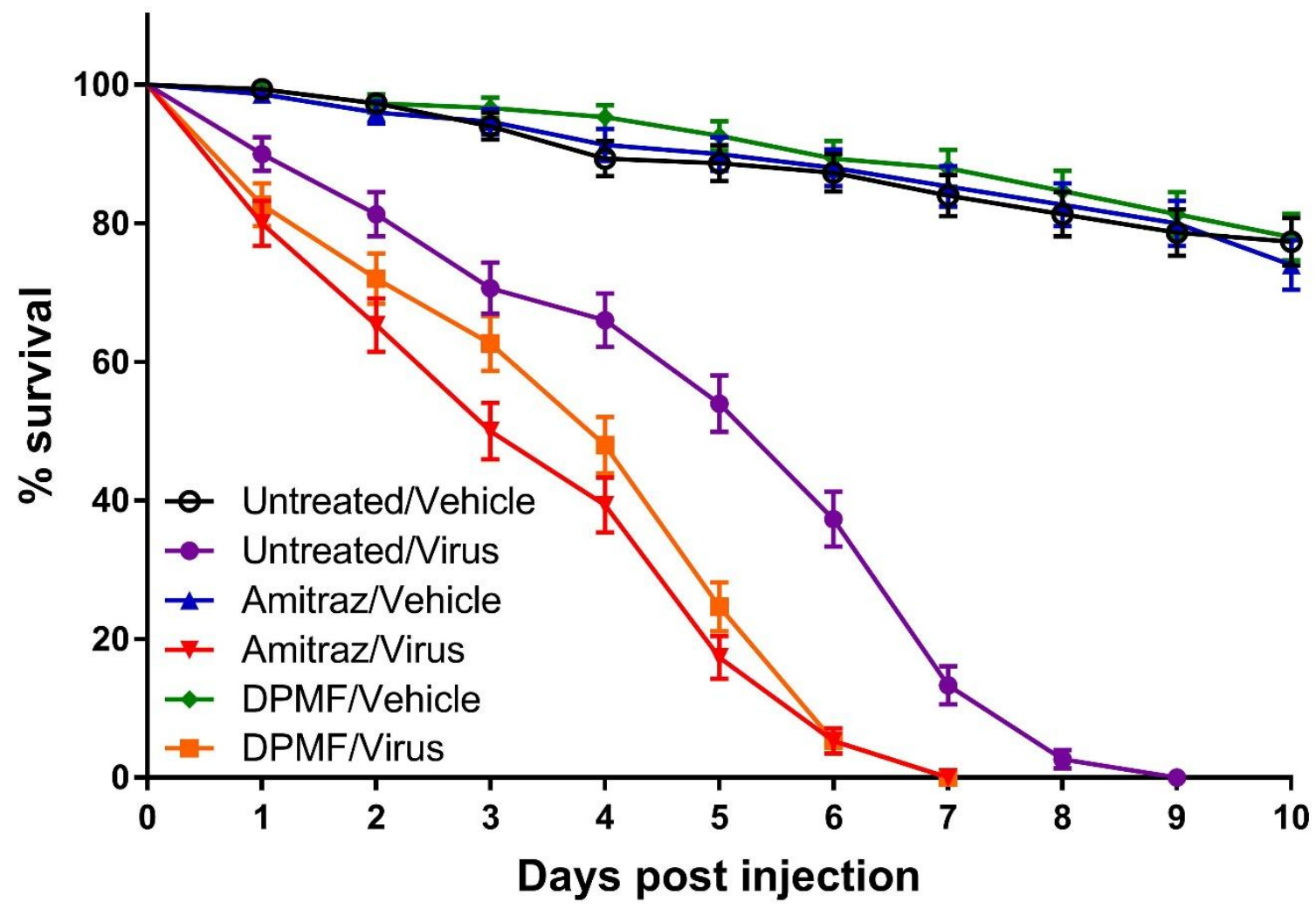

Figure 3. Effect of orally-dosed amitraz and its metabolite DPMF on honey bee survival following a virus challenge. Data presented as Kaplan-Meier survival curves with points representing mean values \pm standard error for 150 bees ( 6 replicate groups of 25 adult bees each per treatment). Amitraz/Virus and DPMF/Virus groups experienced significantly higher mortality than Control/Virus group (Kaplan-Meier logrank test; $P<0.0001)$. Data were analyzed using GraphPad Prism 7 software. 


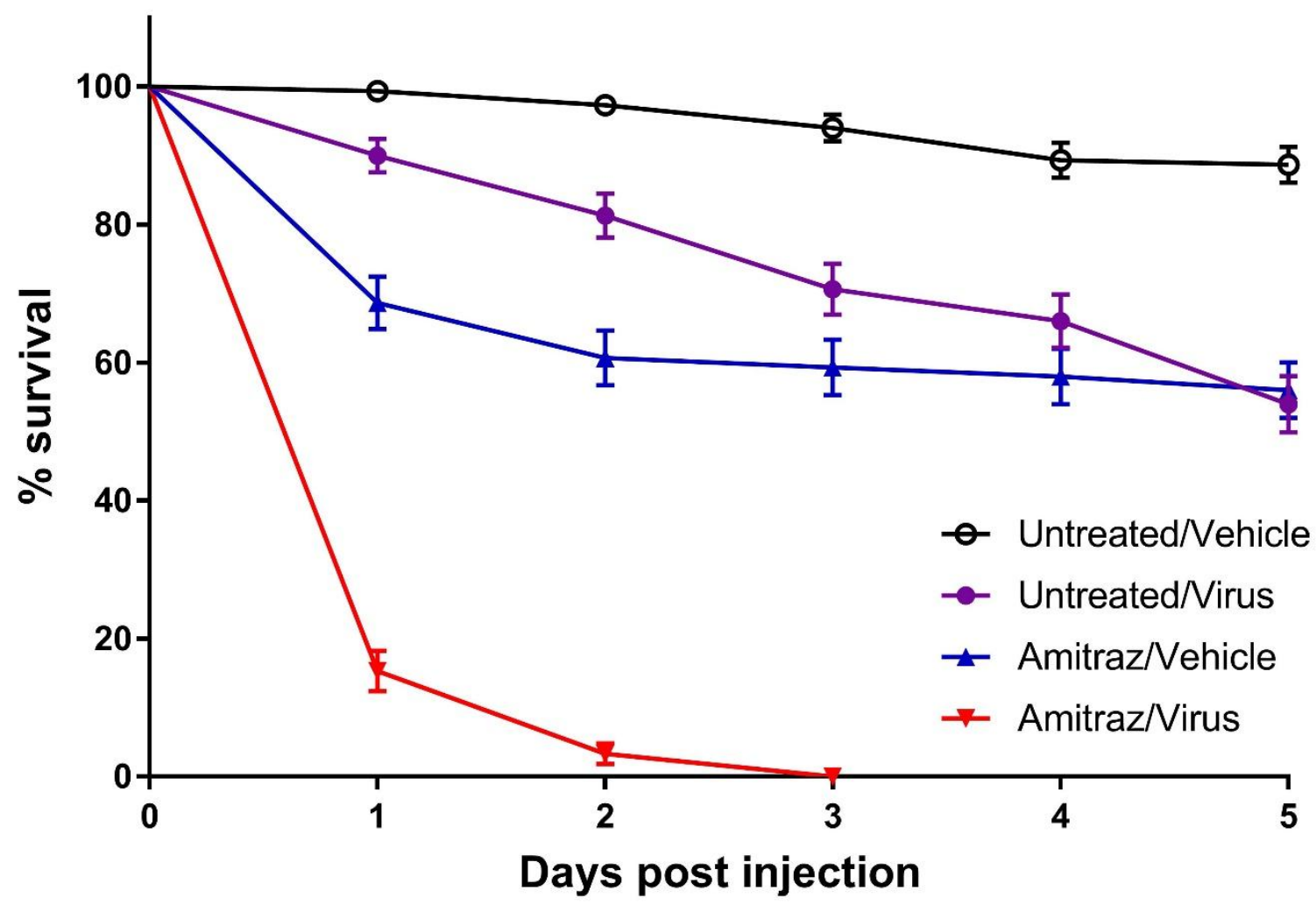

Figure 4. Effect of exposure to Apivar ${ }^{\circledR}$ (amitraz) strips on honey bee survival following a virus challenge. Data presented as Kaplan-Meier survival curves with points representing mean values \pm standard error for 150 bees ( 6 replicate groups of 25 adult bees each per treatment). The Amitraz/Virus group experienced significantly higher mortality than all other groups, while Amitraz/Vehicle and Control/Virus did not differ from one another, but both experienced greater mortality than Control/Vehicle (Kaplan-Meier log-rank test; $P<0.0001$ ). Data were analyzed using GraphPad Prism 7 software. 
Amitraz decreases survival of infected bees

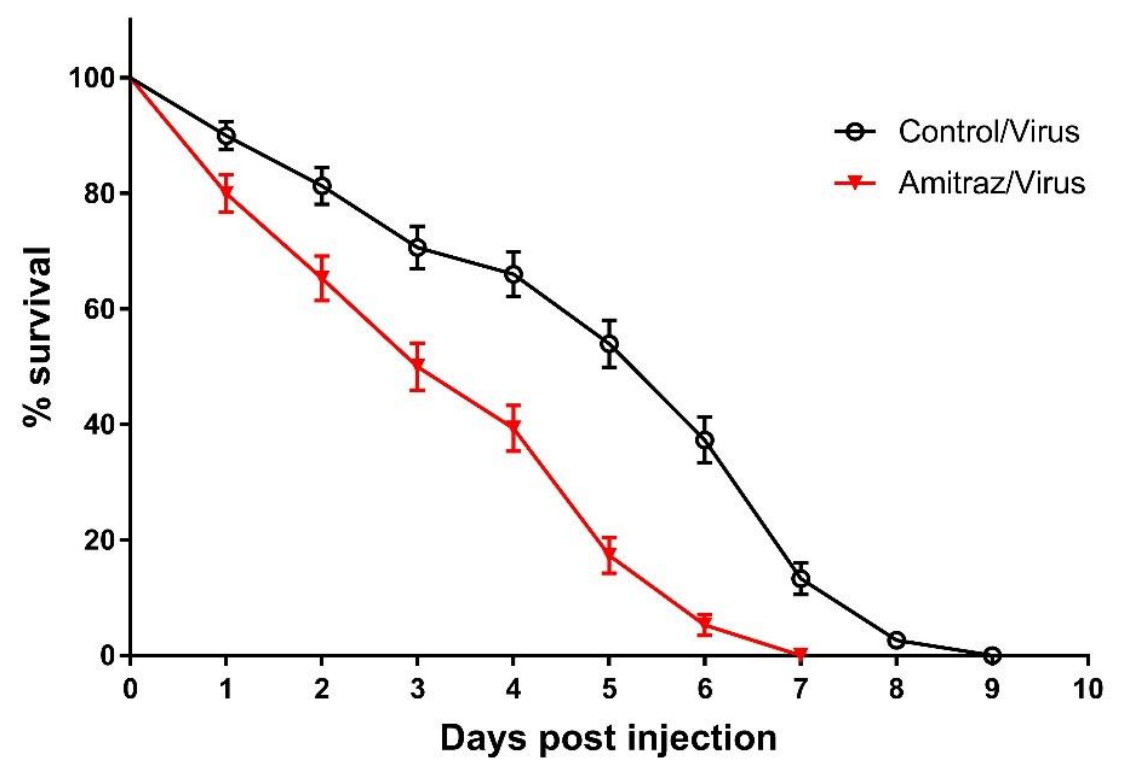

Phentolamine blocks octopaminergic cardioacceleration

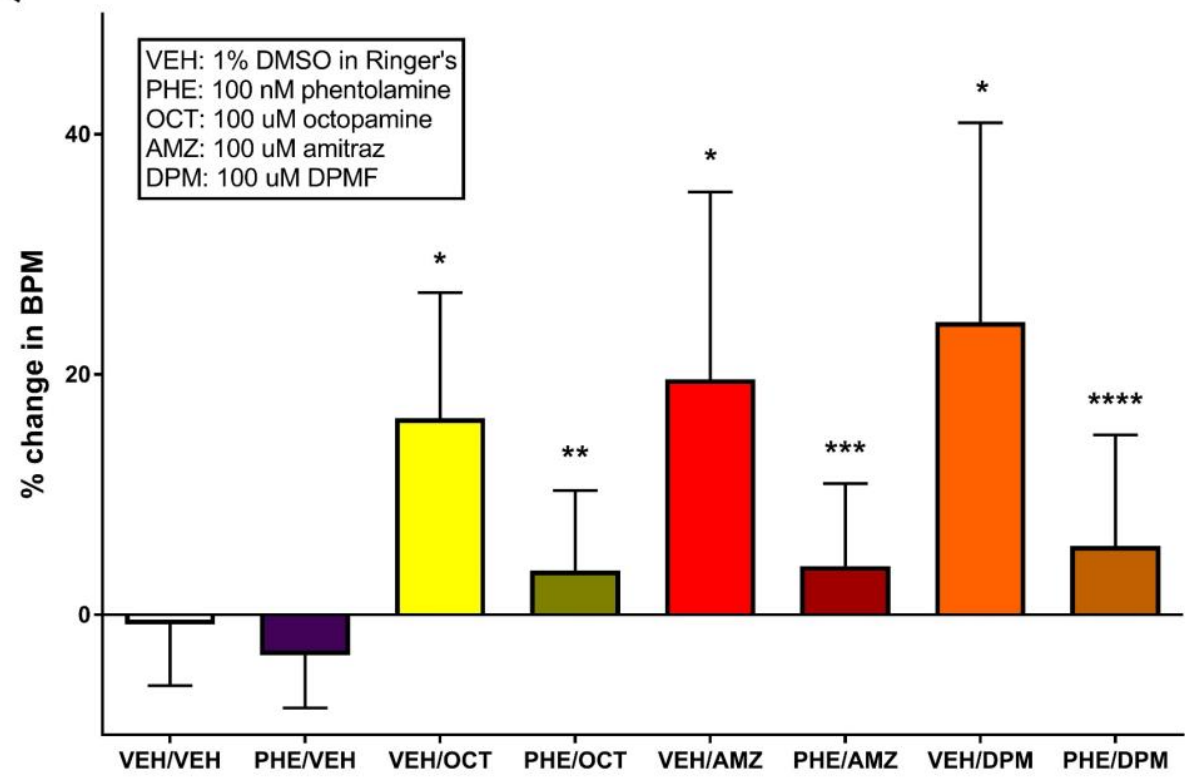

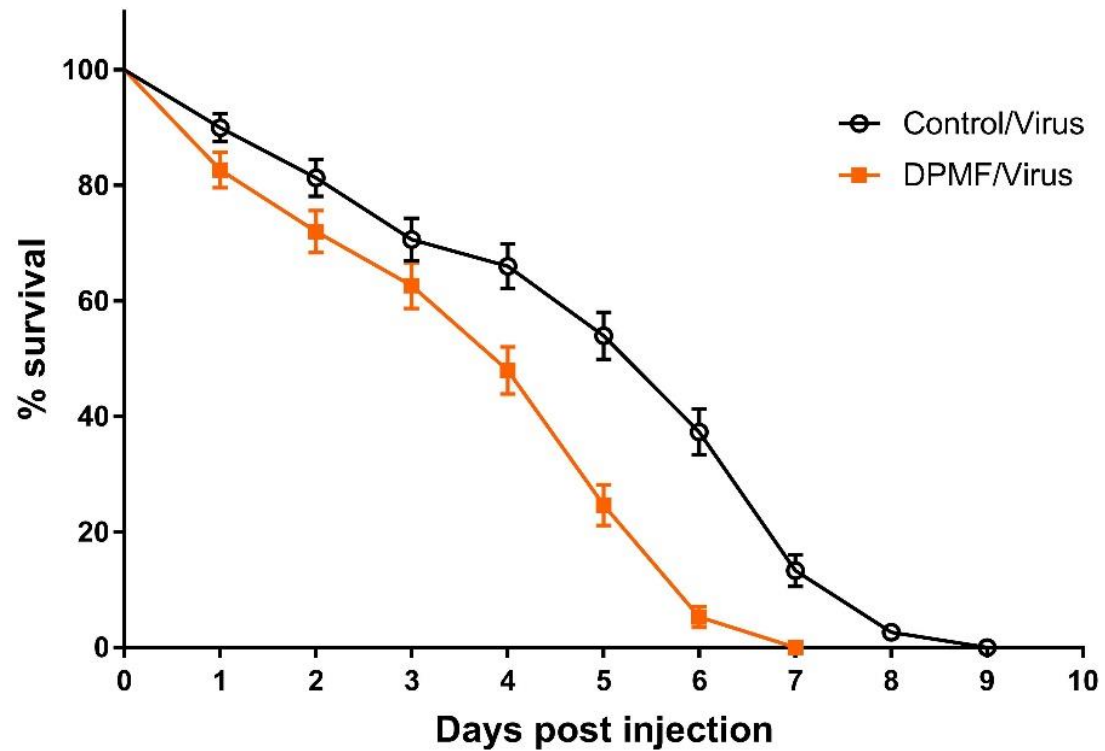

$\overbrace{N_{H}^{-}}^{\mathrm{H}_{3} \mathrm{CH}} \mathrm{CH}_{3}$

DPMF<smiles>NCC(O)c1ccc(O)cc1</smiles>

Octopamine 